EPJ Web of Conferences 73, 06001 (2014)

DOI: $10.1051 /$ epjconf/20147306001

(C) Owned by the authors, published by EDP Sciences, 2014

\title{
Hadronic parity violation in few-nucleon systems
}

\author{
M.R. Schindler ${ }^{\mathrm{a}}$ \\ Department of Physics and Astronomy, University of South Carolina, Columbia, SC 29208, USA
}

\begin{abstract}
The weak interaction between quarks induces a parity-violating component in the interactions between nucleons, which is expected to be suppressed by a factor of about $10^{-7}$ to $10^{-6}$ compared to the dominant parity-conserving part of the interaction. A theoretical framework to analyze and interpret parity-violating observables in few-nucleon systems is described and selected results are presented.
\end{abstract}

\section{Introduction}

Interactions between nucleons are dominated by strong and electromagnetic effects, which conserve parity. However, there is also a parity-violating (PV) component to nucleon-nucleon interactions, which is expected to be suppressed by a factor of roughly $10^{-7}$ to $10^{-6}$ compared to the parity-conserving (PC) component. The PV part of the nucleon interactions stems from an interplay of weak interactions between quarks and the strong interactions that confine the quarks into nucleons. Due to the short range of the weak interaction, it is sensitive to short-range correlations between quarks and can serve as a probe of nonperturbative QCD. For reviews see, e.g., Refs. [1-4]. Parity-violating effects can be isolated in pseudoscalar observables, which would vanish if parity was conserved. Such observables include longitudinal and angular asymmetries, as well as induced polarizations in capture, break-up, and transmission experiments. While PV effects can be enhanced by several orders of magnitude in heavier systems (see, e.g., Ref. [5]), the interpretation of these results is complicated by nuclear structure effects. With the continuing development of high-intensity, low-energy neutron and photon sources, focus has shifted to measurements of PV effects in few-nucleon systems, for which analyses in terms of two- and three-nucleon forces are feasible. In the following, I describe an approach to hadronic parity violation based on effective field theory (EFT) and present selected results from two- and three-nucleon systems.

\section{Hadronic parity violation in pionless EFT}

The energies in many of the few-nucleon experiments aimed at studying hadronic parity violation are much lower than the pion mass. At these very low energies, it is possible to formulate an EFT in which the only dynamical degrees of freedom are nucleons, while pions and all other hadrons are integrated out. The resulting "pionless EFT" $(\operatorname{EFT}(\not))$ has been very successful in describing lowenergy observables, see, e.g., Refs. [6-8] for reviews. The leading-order (LO) Lagrangian describing PV

\footnotetext{
ae-mail: mschindl@mailbox.sc.edu
}

This is an Open Access article distributed under the terms of the Creative Commons Attribution License 4.0, which permits unrestricted use, distribution, and reproduction in any medium, provided the original work is properly cited. 
two-nucleon interactions contains five independent terms and, in the formalism including auxiliary dibaryon fields for S-wave NN states, is given by [9]

$$
\begin{aligned}
\mathcal{L}_{P V}= & -\left[g^{\left({ }^{3} S_{1}-{ }^{1} P_{1}\right)} d_{t}^{i \dagger}\left(N^{T} \sigma_{2} \tau_{2} i \stackrel{\leftrightarrow}{D}_{i} N\right)+g_{(\Delta I=0)}^{\left({ }^{1} S_{0}-{ }^{3} P_{0}\right)} d_{s}^{A \dagger}\left(N^{T} \sigma_{2} \sigma_{i} \tau_{2} \tau_{A} i \stackrel{\leftrightarrow}{D}_{i} N\right)\right. \\
& +g_{(\Delta I=1)}^{\left({ }^{1} S_{0}-{ }^{3} P_{0}\right)} \epsilon^{3 A B} d_{s}^{A \dagger}\left(N^{T} \sigma_{2} \sigma_{i} \tau_{2} \tau^{B} \stackrel{\leftrightarrow}{D}_{i} N\right)+g_{(\Delta I=2)}^{\left({ }^{1} S_{0}-{ }^{3} P_{0}\right)} \mathcal{I}^{A B} d_{s}^{A \dagger}\left(N^{T} \sigma_{2} \sigma_{i} \tau_{2} \tau^{B} i \stackrel{\leftrightarrow}{D}_{i} N\right) \\
& \left.+g^{\left({ }^{3} S_{1}-{ }^{3} P_{1}\right)} \epsilon^{i j k} d_{t}^{i \dagger}\left(N^{T} \sigma_{2} \sigma^{k} \tau_{2} \tau_{3} \stackrel{\leftrightarrow}{D}{ }^{j} N\right)\right]+ \text { h.c. }+\ldots,
\end{aligned}
$$

where $a \mathcal{O} \stackrel{\leftrightarrow}{D}_{i} b=a \mathcal{O} D_{i} b-\left(D_{i} a\right) \mathcal{O} b$, with $\mathcal{O}$ some spin-isospin-operator, and $\mathcal{I}=\operatorname{diag}(1,1,-2)$. The five low-energy constants (LECs) $g^{(X-Y)}$ encode contributions from the hadrons that have been integrated out. In principle, they are calculable from the underlying Standard Model which, however, involves calculations in the non-perturbative regime of QCD. For a preliminary determination of a corresponding LEC in a theory containing explicit pion degrees of freedom see Ref. [10]. It is currently more feasible to determine the values of the LECs by comparison with experimental results.

The Lagrangian of Eq. (1) has been used to determine a number of observables in two- and threenucleon systems. For the results presented in the following sections, the conventions of Ref. [11] are used.

\section{Two-nucleon systems}

Parity-violating effects can be isolated in the scattering of polarized nucleons off an unpolarized nucleon target by considering the longitudinal asymmetry

$$
A_{L}=\frac{\sigma_{+}-\sigma_{-}}{\sigma_{+}+\sigma_{-}},
$$

where $\sigma_{ \pm}$is the total cross section for the scattering of nucleons with helicity \pm 1 . The LO results in $\operatorname{EFT}(\hbar)$ are [12-14]

$$
\begin{gathered}
A_{L}^{n n}=-\sqrt{\frac{32 M}{\pi}} p\left(g_{(\Delta I=0)}^{\left({ }^{1} S_{0}-{ }^{3} P_{0}\right)}-g_{(\Delta I=1)}^{\left({ }^{1} S_{0}-{ }^{3} P_{0}\right)}+g_{(\Delta I=2)}^{\left({ }^{1} S_{0}-{ }^{3} P_{0}\right)}\right), \\
A_{L}^{p p}=-\sqrt{\frac{32 M}{\pi}} p\left(g_{(\Delta I=0)}^{\left({ }^{1} S_{0}-3 P_{0}\right)}+g_{(\Delta I=1)}^{\left({ }^{1} S_{0}-{ }^{3} P_{0}\right)}+g_{(\Delta I=2)}^{\left({ }^{1} S_{0}-{ }^{3} P_{0}\right)}\right), \\
A_{L}^{n p}=-\sqrt{\frac{32 M}{\pi}} p \frac{\frac{d \sigma^{1} S_{0}}{d \Omega}}{\frac{d \sigma^{1} S_{0}}{d \Omega}+3 \frac{d \sigma^{3} S_{1}}{d \Omega}}\left(g_{(\Delta I=0)}^{\left({ }^{1} S_{0}-{ }^{3} P_{0}\right)}-2 g_{(\Delta I=2)}^{\left({ }^{1} S_{0}-{ }^{3} P_{0}\right)}\right) \\
-\sqrt{\frac{32 M}{\pi}} p \frac{\frac{d \sigma^{3} S_{1}}{d \Omega}}{\frac{d \sigma^{1} S_{0}}{d \Omega}+3 \frac{d \sigma^{3} S_{1}}{d \Omega}}\left(g^{\left({ }^{3} S_{1}-{ }^{1} P_{1}\right)}+2 g^{\left({ }^{3} S_{1}-{ }^{3} P_{1}\right)}\right),
\end{gathered}
$$

where $M$ is the nucleon mass and $p$ the nucleon momentum in the center-of-mass frame. The $p p$ result does not contain Coulomb effects, which have been shown to contribute approximately $3 \%$ for the energies and angular ranges that have been considered experimentally [14].

The $n p$ asymmetry is related to a different observable, the spin rotation angle per unit length in the transmission of a polarized neutron beam through an unpolarized hydrogen target. Up to next-to-leading 
(NLO) order it is given by [11]

$$
\frac{1}{\rho} \frac{d \phi_{\mathrm{PV}}^{n p}}{d l}=4 \sqrt{2 \pi M}\left(\frac{2 g^{\left({ }^{3} S_{1}-{ }^{3} P_{1}\right)}+g^{\left({ }^{3} S_{1}-{ }^{1} P_{1}\right)}}{\gamma_{t}} \frac{Z_{t}+1}{2}+\frac{g_{(\Delta I=0)}^{\left({ }^{1} S_{0}-{ }^{3} P_{0}\right)}-2 g_{(\Delta I=2)}^{\left({ }^{1} S_{0}-{ }^{3} P_{0}\right)}}{\gamma_{s}} \frac{Z_{s}+1}{2}\right),
$$

where $\rho$ is the target density, $\gamma_{t / s}$ are the poles in the NN scattering amplitudes in the ${ }^{3} S_{1}$ and ${ }^{1} S_{0}$ channels, respectively, and $Z_{t / s}=\frac{1}{1-\gamma_{t, s} r_{t / s}}$, with $r_{t / s}$ the effective ranges in the corresponding channels.

The angular asymmetry $A_{\gamma}$ in polarized neutron capture, $\vec{n} p \rightarrow d \gamma$, is currently being measured at the Spallation Neutron Source at Oak Ridge National Laboratory [15]. The LO EFT $(\not)$ result is given by $[9,16]$

$$
A_{\gamma}=\frac{4}{3} \sqrt{\frac{2}{\pi}} \frac{M^{\frac{3}{2}}}{\kappa_{1}\left(1-\gamma_{t} a_{s}\right)} g^{\left({ }^{3} S_{1}-{ }^{3} P_{1}\right)},
$$

where $\kappa_{1}$ is the anomalous nucleon isovector magnetic moment and $a_{s}$ the scattering length in the ${ }^{1} S_{0}$ channel.

Complementary information on the PV couplings can be obtained from the circular photon polarization in unpolarized neutron capture, $n p \rightarrow d \vec{\gamma}$, which in $\operatorname{EFT}(\not t)$ is given by [9]

$$
P_{\gamma}=-2 \sqrt{\frac{2}{\pi}} \frac{M^{\frac{3}{2}}}{\kappa_{1}\left(1-\gamma a_{S}\right)}\left[\left(1-\frac{2}{3} \gamma a_{s}\right) g^{\left({ }^{3} S_{1}-{ }^{1} P_{1}\right)}+\frac{\gamma a_{s}}{3}\left(g_{(\Delta I=0)}^{\left({ }^{1} S_{0}-{ }^{3} P_{0}\right)}-2 g_{(\Delta I=2)}^{\left({ }^{1} S_{0}-3 P_{0}\right)}\right)\right] .
$$

For exactly reversed kinematics, $P_{\gamma}$ is equal to the asymmetry $A_{L}^{\gamma}$ in the reaction $\vec{\gamma} d \rightarrow n p$. A measurement of $A_{L}^{\gamma}$ is being considered as a flagship experiment for a possible upgrade of the HIGS facility at the Triangle Universities Nuclear Laboratory.

\section{Three-nucleon systems}

In the PC sector of $\operatorname{EFT}(\not)$, three-nucleon interactions are enhanced compared to an estimate of their importance based on power counting [17, 18]. In Ref. [19], it was shown that such an enhancement does not occur for PV three-nucleon interactions and that they do not contribute at LO and NLO. Therefore, up to an accuracy of roughly $10 \%$, few-nucleon observables can be described by PV twonucleon interactions alone. Numerical support for these theoretical considerations is given by the $n d$ scattering results of Refs. $[11,20]$. These results can be related to the neutron spin rotation angle in a deuteron target, which to NLO is given by [11]

$$
\frac{1}{\rho} \frac{d \phi_{\mathrm{PV}}^{n d}}{d l}=\left([16 \pm 1.6] g^{\left({ }^{(} S_{1}-{ }^{1} P_{1}\right)}+[34 \pm 3.4] g^{\left({ }^{3} S_{1}-{ }^{3} P_{1}\right)}+[4.6 \pm 1.0]\left(3 g_{(\Delta I=0)}^{\left({ }^{1} S_{0}-{ }^{3} P_{0}\right)}-2 g_{(\Delta I=1)}^{\left({ }^{(} S_{0}-{ }^{3} P_{0}\right)}\right)\right) \frac{\mathrm{rad}}{\mathrm{MeV}^{\frac{1}{2}}},
$$

including theoretical error estimates.

\section{Conclusions and outlook}

Hadronic parity violation originates in an interplay of weak and strong interactions at low energies, and thus provides a unique probe of nonperturbative QCD. EFT $(\pi)$ provides a unified framework for describing PC and PV interactions as well as external currents. The short-distance details of parity-violating effects are encoded in five low-energy constants, which can be determined given a sufficient number of experimental results. The calculations presented here form part of a comprehensive effort to analyze and interpret hadronic parity violation in few-nucleon systems. There are a number of further observables in four- and five-nucleon systems, such as an asymmetry in $\vec{p}^{4} \mathrm{He}$ scattering 
or the neutron spin rotation angle in a ${ }^{4} \mathrm{He}$ target, for which experimental results exist [21, 22]. Consistent EFT calculations for these observables are very desirable and would provide important additional information.

I thank the organizers for putting together such an interesting conference. I am also grateful to H.W. Grießhammer, D.R. Phillips, and R.P. Springer for their collaboration and many stimulating discussions. This work was supported by the US Department of Energy under grant no. DE-SC0010300.

\section{References}

[1] E.G. Adelberger, W.C. Haxton, Ann. Rev. Nucl. Part. Sci. 35, 501 (1985)

[2] M.J. Ramsey-Musolf, S.A. Page, Ann. Rev. Nucl. Part. Sci. 56, 1 (2006), hep-ph/0601127

[3] W. Haxton, B. Holstein, Prog. Part. Nucl. Phys. 71, 185 (2013), 1303. 4132

[4] M. Schindler, R. Springer, Prog. Part. Nucl. Phys. 72, 1 (2013), 1305.4190

[5] C. Bowman, J. Bowman, V. Yuan, Phys. Rev. C 39, 1721 (1989)

[6] S.R. Beane, P.F. Bedaque, W.C. Haxton, D.R. Phillips, M.J. Savage, In *Shifman, M. (ed.): At the frontier of particle physics, vol. 1* pp. 133-269. (2000), nucl-th/0008064

[7] P.F. Bedaque, U. van Kolck, Ann. Rev. Nucl. Part. Sci. 52, 339 (2002), nucl-th/0203055

[8] L. Platter, Few Body Syst. 46, 139 (2009), 0904.2227

[9] M.R. Schindler, R.P. Springer, Nucl. Phys. A 846, 51 (2010), 0907.5358

[10] J. Wasem, Phys. Rev. C 85, 022501 (2012), 1108.1151

[11] H.W. Grießhammer, M.R. Schindler, R.P. Springer, Eur. Phys. J. A 48, 7 (2012), 1109. 5667

[12] S.L. Zhu, C.M. Maekawa, B.R. Holstein, M.J. Ramsey-Musolf, U. van Kolck, Nucl. Phys. A 748, 435 (2005), nucl-th/0407087

[13] B.R. Holstein, Fizika. B 14, 165 (2005), nucl-th/0607038

[14] D.R. Phillips, M.R. Schindler, R.P. Springer, Nucl. Phys. A 822, 1 (2009), 0812. 2073

[15] M. Gericke, R. Alarcon, S. Balascuta, L. Barron-Palos, C. Blessinger et al., Phys. Rev. C 83, 015505 (2011)

[16] M.J. Savage, Nucl. Phys. A 695, 365 (2001), nucl-th/0012043

[17] P.F. Bedaque, H.W. Hammer, U. van Kolck, Phys. Rev. Lett. 82, 463 (1999), nucl-th/9809025

[18] P.F. Bedaque, H.W. Hammer, U. van Kolck, Nucl. Phys. A 676, 357 (2000), nucl-th/9906032

[19] H.W. Grießhammer, M.R. Schindler, Eur. Phys. J. A 46, 73 (2010), 1007. 0734

[20] J. Vanasse, Phys. Rev. C 86, 014001 (2012), 1110.1039

[21] J. Lang, T. Maier, R. Muller, F. Nessi-Tedaldi, T. Roser et al., Phys. Rev. Lett. 54, 170 (1985)

[22] W. Snow, C. Bass, T. Bass, B. Crawford, K. Gan et al., Phys. Rev. C 83, 022501 (2011) 